\title{
The Association Between Ideal Cardiovascular Health and Health-Related Quality of Life in Adults: A Population-Based Cross-Sectional Study
}

\begin{abstract}
Xueyan Wu ${ }^{1}$ Xiaotian Liu ${ }^{1}$ Wei Liao ${ }^{1}$ Ning Kang ${ }^{1}$ Shengxiang Sang ${ }^{1}$ Tanko Abdulai ${ }^{1}$ Miaomiao Niu ${ }^{1}$ Yaling $\mathrm{He}^{1}$ Zhihan Zhai ${ }^{1}$ Mingming Pan ${ }^{1}$ Chongjian Wang ${ }^{1}$ and Yuqian $\mathrm{Li}^{2 *}$

${ }^{1}$ Department of Epidemiology and Biostatistics, College of Public Health, Zhengzhou University, Zhengzhou, China, ${ }^{2}$ Department of Clinical Pharmacology, School of Pharmaceutical Science, Zhengzhou University, Zhengzhou, China
\end{abstract}

Objectives: The study aimed to explore the association between Ideal cardiovascular health $(\mathrm{ICH})$ and health-related quality of life (HRQoL) using the European Quality of Life Five Dimension Five Level Scale (EQ-5D-5L) among rural population.

Methods: This study included 20,683 participants aged 18-79 years from "the Henan Rural Cohort study". Generalized linear and Tobit regression models were employed to explore the associations of $\mathrm{ICH}$ with EQ-5D-5L utility scores and visual analogue scale (VAS) scores, respectively.

Results: The mean EQ-5D-5L utility scores and VAS scores were $0.962 \pm 0.095$ and $79.52 \pm 14.02$, respectively. Comparing with poor CVH participants (EQ-5D-5L utility scores and VAS scores: $0.954 \pm 0.111$ and $78.44 \pm 14.29$ ), people with intermediate and ideal $\mathrm{CVH}$ had higher EQ-5D-5L utility scores $(0.969 \pm 0.079$ and $0.959 \pm 0.099)$ and VAS scores $(80.43 \pm 13.65$ and $79.28 \pm 14.14)$. ICH scores were positively correlated with EQ$5 \mathrm{D}-5 \mathrm{~L}$ utility scores $(0.007(0.004,0.009))$ and VAS scores $(0.295(0.143,0.446))$, respectively.

Conclusions: Higher $\mathrm{ICH}$ scores is positive associated with better HRQoL in rural population, which suggests that improvement of cardiovascular health may help to enhance HRQoL among rural population.

Received: 06 August 2020 Accepted: 02 February 2021

Published: 19 March 2021

Citation:

Wu X Liu X Liao W Kang N Sang S Abdulai T Niu M He Y Zhai Z Pan M

Wang $C$ and $L i Y$ (2021) The

Association Between Ideal Cardiovascular Health and HealthRelated Quality of Life in Adults: A

Population-Based Cross-

Sectional Study.

Int J Public Health 66:592043.

doi: 10.3389/ijph.2021.592043
Keywords: ideal cardiovascular health, health-related quality of life, EQ-5D-5L, rural population, Ideal health behaviors, Ideal health factors

\section{INTRODUCTION}

Cardiovascular disease (CVD) is now the leading cause of premature mortality and disability in world [1, 2]. In 2010, ideal cardiovascular health (ICH) was formulated by the American Heart Association to reduce deaths from all CVDs [3]. ICH was defined with four ideal health behaviors (ideal smoking status, ideal body mass index (BMI), ideal physical activity, and ideal diet) and four ideal health factors (ideal smoking status, ideal total cholesterol (TC), ideal blood pressure (BP), and ideal fasting plasma glucose (FPG)) [3]. Prospective studies consistently indicated that individuals 
with a higher number of ideal cardiovascular health metrics have lower risks of hypertension [4], type 2 diabetes mellitus [5], CVD events [6, 7], and all-cause mortality [4].

Health-related quality of life (HRQoL) refers to the individual's health status under the influence of illness and injury, medical intervention, aging and social environment change, as well as the subjective satisfaction associated with its economic, cultural background and value orientation. It is a multidimensional concept which was main contented by health status and subjective satisfaction [8]. The European Quality of Life Five Dimension Five Level Scale (EQ-5D-5L) is one of the simplest and most commonly used self-reported instruments to evaluate HRQoL due to its simple, low response requirements, and generally high acceptance [9].

Prior literature has demonstrated an association of individual cardiovascular risk factors with HRQoL $[10,11]$. However, most studies considered single cardiovascular risk factors. Although some previous studies also have reported the association between cardiovascular health and HRQoL [12-14]. These studies were conducted in western countries and used the Medical Outcomes Study 12-Item Short Form Health Survey (SF-12) and HRQoL-4 tool. Hence, the aim of this study was to explore the association between ICH and HRQoL using EQ-5D-5L in Chinese rural population.

\section{METHODS}

\section{Study Design and Participants}

The Henan Rural Cohort Study is a prospective study of chronic non-communicable diseases among a large sample of rural people established in Henan Province, China, from 2015 to 2017. In short, the cohort recruited participants aged from 18 to 79 by multi-stage stratified cluster sampling. 39,259 people were included in the cohort study, with a response rate of $93.7 \%$. The details of this cohort have been described elsewhere [15]. In this study, 23,510 participants with complete information on EQ5D-5L were included. Then, the participants were further excluded if they [1]: were diagnosed with coronary heart disease $(n=1,247)$ [2]; were diagnosed with stroke $(n=1710)$ [3]; were missed information needed in the present study $(\mathrm{n}=$ 78). Finally, 20,683 adults were ultimately included in the current study.

This study was consistent with "Zhengzhou University Life Science Ethics Committee" [Ethics approval code [2015] MEC (S128)]. Participants were required to provide informed consent.

\section{Data Collection}

A standard questionnaire was administered by face to face interviews, including demographic characteristics (age, gender, educational level, marital status, and per capita monthly income), lifestyles (smoking, drinking, and physical activity), behaviors, dietary patterns (through Food Frequency Questionnaire (FFQ)), individual history of diseases and medication use were collected. The International Physical Activity Questionnaire (IPAQ 2001) was used to assess the levels of physical activity (16). The
FFQ based on the Dietary Guidelines for Chinese Residents and the eating habits of Henan people. Previous studies have shown that the FFQ has good reproducibility and validity (17). Individual histories of disease and medication were selfreported by the participants. The height and weight of participants were measured twice, and the average readings were computed to analyze. Body mass index (BMI, $\mathrm{kg} / \mathrm{m}^{2}$ ) was calculated as weight $(\mathrm{kg})$ divided the square of height $(\mathrm{m})$. Blood pressure was measured three times by electronic sphygmomanometer in the right arm in a sitting position after at least $5 \mathrm{~min}$ rest. There were 30 s intervals between the three measurements. Venous blood samples were collected from subjects after an overnight fast of at least 8 hours and stored in $-80^{\circ} \mathrm{C}$ cryogenic refrigerator before analysis. The FBG and TC were measured by Roche Cobas C501 automatic biochemical analyzer. The details of the equipment for anthropometric and clinical examinations have been introduced elsewhere (15).

\section{Ideal Cardiovascular Health Metrics}

ICH metrics were as following according to the AHA (3) : ideal smoking status, never a smoker; ideal BMI, BMI $<25 \mathrm{~kg} / \mathrm{m}^{2}$; ideal physical activity, physical activity $\geq 150 \mathrm{~min} / \mathrm{wk}$ of moderate intensity or $\geq 75 \mathrm{~min} / \mathrm{wk}$ of vigorous intensity or $\geq 150 \mathrm{~min} / \mathrm{wk}$ of moderate-vigorous intensity combination; ideal diet, $\geq 4$ components; ideal TC, TC $<5.18 \mathrm{mmol} / \mathrm{L}$ untreated; ideal BP, SBP $<120 / \mathrm{DBP}<80 \mathrm{~mm} \mathrm{Hg}$ untreated, and ideal FPG, FPG $<5.6 \mathrm{mmol} / \mathrm{L}$ untreated. (3) Furthermore, ICH was defined as follows: the simultaneous presence of 4 IHB (ideal smoking status, ideal BMI, ideal PA, and ideal diet) and 4 IHF (ideal smoking status, ideal TC, ideal BP, and ideal FPG) in the absence of a history of cardiovascular disease (3). We made some adaptations as appropriate for the healthy diet score. Ideal diet was defined as healthy diet score $\geq 4$ components (18), including fruits and vegetables $\geq 500 \mathrm{~g} / \mathrm{d}$, fish $\geq 200 \mathrm{~g} /$ week, soybean products $\geq 125 \mathrm{~g} / \mathrm{d}$, red meat $<75 \mathrm{~g} /$ $\mathrm{d}$, and drinking tea.

\section{EQ-5D-5L}

In this study, the EQ-5D-5L was utilized to evaluate the HRQoL of participants. The five dimensions of EQ-5D-5L were consisted of mobility (MO), self-care (SC), usual activities (UA), pain/ discomfort (PD) and anxiety/depression (AD). Each dimension had five levers including no problems, slight problems, moderate problems, severe problems and extreme problems. For example, participants were asked if they had any problems walking. If they did, they were asked if it was serious and if they did not, they were asked if it was a slight or a moderate problem. Participants that reported their problems as serious were asked if they could walk at that moment. If they could, they were classified as having severe problems walking and if they could not, they were classified as having extreme problems.

The EQ-5D utility scores were calculated based on the recently available Chinese value set for the EQ-5D-5L instrument [19]. Utility $=1-\mathrm{MO} \times \mathrm{Ln}-\mathrm{SC} \times \mathrm{Ln}-\mathrm{UA} \times \mathrm{Ln}-\mathrm{PD} \times \mathrm{Ln}-\mathrm{AD} \times \mathrm{Ln}$ $(\mathrm{n}=1,2,3,4,5)$. Scores ranged from -0.200 to 1.000 , with one representing full health, 0 representing death, and a score $<0$ 
TABLE 1 | Characteristics of the participants (Collected during 2015-2017, China).

\begin{tabular}{|c|c|c|c|c|c|}
\hline Variables & Total & Poor CVH & Intermediate CVH & Ideal CVH & $p$ \\
\hline Sex & & & & & $<0.001$ \\
\hline Women & $12,348(59.70)$ & $1,289(37.49)$ & $6,039(57.96)$ & $5,020(73.55)$ & \\
\hline Marital status & & & & & 0.060 \\
\hline Married/cohabiting & $18,753(90.67)$ & 3,127 (90.95) & $9,399(90.20)$ & $6,227(91.24)$ & \\
\hline Unmarried/divorced/widowed & $1930(9.33)$ & $311(9.05)$ & $1,021(9.80)$ & $598(8.76)$ & \\
\hline Junior high school & 8,085 (39.09) & $1,322(38.45)$ & $3,996(38.35)$ & 2,767 (40.54) & \\
\hline High school or above & $4,102(19.83)$ & 733 (21.32) & $1833(17.59)$ & $1,536(22.51)$ & \\
\hline Income (RMB per month) & & & & & $<0.001$ \\
\hline$<500$ & $7,433(35.94)$ & 1,148 (33.39) & $3,891(37.34)$ & 2,394 (35.08) & \\
\hline $500 \sim$ & $6,599(31.9)$ & $1,150(33.45)$ & $3,378(32.42)$ & $2071(30.34)$ & \\
\hline $1,000 \sim$ & $6,651(32.16)$ & $1,140(33.16)$ & $3,151(30.24)$ & $2,360(34.58)$ & \\
\hline No drinking & $16,004(77.38)$ & $2061(59.95)$ & $8,006(76.83)$ & 5,937 (86.99) & \\
\hline Current drinking & $4,679(22.62)$ & $1,377(40.05)$ & $2,414(23.17)$ & 869 (13.01) & \\
\hline $\mathrm{BMI}\left(\mathrm{kg} / \mathrm{m}^{2}\right.$ mean $\left.\pm \mathrm{SD}\right)$ & $24.93 \pm 3.60$ & $27.60 \pm 3.13$ & $25.44 \pm 3.46$ & $22.82 \pm 2.74$ & $<0.001$ \\
\hline Utility scores, mean (SD) & $0.962 \pm 0.095$ & $0.954 \pm 0.111$ & $0.959 \pm 0.099$ & $0.969 \pm 0.079$ & $<0.001$ \\
\hline VAS scores, mean (SD) & $79.52 \pm 14.02$ & $78.44 \pm 14.29$ & $79.28 \pm 14.14$ & $80.43 \pm 13.65$ & $<0.001$ \\
\hline Total & $20,863(100.00)$ & $3,438(16.62)$ & $10,420(50.38)$ & $6,825(33.00)$ & \\
\hline
\end{tabular}

SD, standard deviation; BMI, body mass index; EQ-5D-5L utility scores: The European Quality of Life Five Dimension Five Level Scale utility scores; VAS scores: Visual analogue scale scores; Poor CVH, poor cardiovascular health ( 0 -2 of ideal cardiovascular health scores); Intermediate CVH, intermediate cardiovascular health (three to four of ideal cardiovascular health scores); Ideal $\mathrm{CVH}$, ideal cardiovascular health (five to seven of ideal cardiovascular health scores).

representing a health status worse than death. The EQ-5D-5L also included a visual analogue scale (VAS) where participants marked their own health on a scale ranging from "best imaginable" (100) to "worst imaginable" state of health (0).

\section{Statistical Analysis}

The continuous data were represented by mean and standard deviation (SD) and the categorical data were tabulated with frequencies and percentages. Student's $t$ test and chi-square test was used to compare the difference between groups.

Due to the distribution of the EQ-5D utility was skewed and the utility score was censored at 1, multivariate Tobit regression model was performed to assess the association between ICH and EQ-5D utility scores [20]. A generalized linear model (GLM) was chosen assess the association between ICH and VAS scores, because the VAS score was abnormal distribution continuous variable. Apart from the linear regression model, we also used a logistic regression model to examine the association between EQ-5D utility scores and VAS scores and the prevalence of ideal CVH (ICH scores $\geq 5$ ). A range of potential confounders were adjusted, including age ( $<40,40-60$ or $\geq 60$ years), gender (men or women), educational level (primary school or illiteracy, junior high school, or high school or above), income (<500, 500-100 or $\geq 1000 \mathrm{RMB}$ per month) and drinking (no drinking or current drinking). Results were expressed as increased ICH scores and 95\% confidence intervals (95\% CIs) or odds ratio of ideal CVH associated with increment of EQ-5D utility scores and VAS scores. The potential modification effects of gender, age, education level, income and drinking were examined by adding an interaction term into the adjusted model. All statistical analyses were performed by SPSS software V.21.0, STATA 15 for Windows and R version 3.6.3.

\section{RESULTS}

\section{Characteristics of the Participants}

A summary of participants' demographic characteristics is shown in Table 1. Of all participants, 6,825 (33.00\%) showed Ideal $\mathrm{CVH}$ ( $\mathrm{ICH}$ scores $\geq 5$ ). Lower mean age and BMI, higher fractions of women and married/cohabiting were observed among those with ideal ICH than others. Participants with ideal CVH tended to be non-smoker and non-drinkers. In different ICH groups, the EQ-5D utility scores and VAS scores of participants with intermediate and ideal $\mathrm{CVH}$ were higher than those poor $\mathrm{CVH}$, indicating that participants with ideal $\mathrm{CVH}$ had higher HRQoL.

\section{Reported Health Problems}

The percentage of respondents who reported problems (no, slight, moderate, severe, or extreme problems) based on the EQ-5D-5L questionnaire is shown in Table 2. The dimension with the highest proportion of patients with self-reported problems was the pain/discomfort dimension (4.23\%), followed by mobility dimension (2.50\%). The self-care 
TABLE 2 | The association between ideal cardiovascular health metrics and health-related quality of life (Collected during 2015-2017, China).

\begin{tabular}{|c|c|c|c|c|c|c|c|}
\hline \multirow{2}{*}{$\frac{\mathrm{ICH} \text { metrics }}{\text { (ref. = non-ideal) }}$} & \multirow{2}{*}{$\begin{array}{c}\text { Total, } \mathbf{n} \\
\text { (\%) }\end{array}$} & \multicolumn{3}{|c|}{ EQ-5D-5L utility scores ${ }^{a}$} & \multicolumn{3}{|c|}{ VAS scores $^{b}$} \\
\hline & & COE & SE & $p$ & $\beta$ & SE & $p$ \\
\hline \multicolumn{8}{|l|}{ Total cholesterol } \\
\hline Ideal & $12,451(60.20)$ & 0.012 & 0.004 & 0.004 & 0.447 & 0.199 & 0.024 \\
\hline \multicolumn{8}{|l|}{ Blood pressure } \\
\hline Ideal & $7,976(38.56)$ & 0.002 & 0.004 & 0.673 & 0.017 & 0.206 & 0.933 \\
\hline \multicolumn{8}{|c|}{ Fasting plasma glucose } \\
\hline Ideal & 14,173 (68.52) & 0.010 & 0.004 & 0.016 & 0.597 & 0.208 & 0.004 \\
\hline \multicolumn{8}{|l|}{ Smoking } \\
\hline Ideal & 14,965 (72.35) & -0.012 & 0.007 & 0.072 & 0.347 & 0.326 & 0.286 \\
\hline \multicolumn{8}{|l|}{ Physical activity } \\
\hline Ideal & 18,602 (89.94) & 0.046 & 0.007 & 0.001 & 1.973 & 0.321 & $<0.001$ \\
\hline \multicolumn{8}{|l|}{$\mathrm{BMI}\left(\mathrm{kg} / \mathrm{m}^{2}\right)$} \\
\hline Ideal & $10,923(52.81)$ & 0.007 & 0.004 & 0.079 & 0.039 & 0.191 & 0.840 \\
\hline \multicolumn{8}{|l|}{ Healthy diet score } \\
\hline Ideal & $105(0.51)$ & -0.010 & 0.029 & 0.734 & 0.363 & 1.343 & 0.787 \\
\hline
\end{tabular}

ICH, Ideal cardiovascular health; BMI, body mass index; EQ-5D-5L utility scores: The European Quality of Life Five Dimension Five Level Scale utility scores; VAS scores: Visual analogue scale scores.

a Multivariate Tobit regression model.

${ }^{b}$ Generalized linear model.

TABLE 3 | The association between ideal cardiovascular health scores and health-related quality of life (Collected during 2015-2017, China).

\begin{tabular}{|c|c|c|c|c|c|c|c|}
\hline \multirow[t]{2}{*}{ Ideal scores } & \multirow{2}{*}{$\begin{array}{c}\text { Total, } \mathbf{n} \\
(\%)\end{array}$} & \multicolumn{3}{|c|}{ Utility scores $^{a}$} & \multicolumn{3}{|c|}{ VAS scores $^{b}$} \\
\hline & & Mean \pm SD & $\begin{array}{c}\text { COE } \\
(95 \% \mathrm{Cl})\end{array}$ & $p$ & Mean \pm SD & $\begin{array}{c}\beta \\
(95 \% \mathrm{Cl})\end{array}$ & $p$ \\
\hline IHB scores & & & $0.012(0.016,0.018)$ & $<0.001$ & & $0.519(0.307,0.892)$ & $<0.001$ \\
\hline 0 & $422(2.04)$ & $0.966 \pm 0.097$ & & & $79.06 \pm 13.47$ & & \\
\hline 1 & $3,063(14.81)$ & $0.961 \pm 0.108$ & & & $79.44 \pm 14.20$ & & \\
\hline 2 & $10,087(48.77)$ & $0.960 \pm 0.097$ & & & $79.50 \pm 14.07$ & & \\
\hline 3 & $7,086(34.26)$ & $0.964 \pm 0.087$ & & & $79.61 \pm 13.92$ & & \\
\hline 4 & $25(0.12)$ & $0.956 \pm 0.070$ & & & $78.52 \pm 13.84$ & & \\
\hline IHF scores & & & $0.005(0.001,0.009)$ & 0.023 & & $0.282(0.088,0.476)$ & 0.004 \\
\hline 0 & 729 (3.25) & $0.968 \pm 0.088$ & & & $79.56 \pm 13.50$ & & \\
\hline 1 & $3,684(17.81)$ & $0.955 \pm 0.106$ & & & $78.34 \pm 14.30$ & & \\
\hline 2 & $6,530(31.57)$ & $0.957 \pm 0.102$ & & & $78.89 \pm 14.22$ & & \\
\hline 3 & 6,139 (39.68) & $0.963 \pm 0.932$ & & & $79.91 \pm 14.02$ & & \\
\hline 4 & $3,601(17.41)$ & $0.973 \pm 0.071$ & & & $81.19 \pm 13.28$ & & \\
\hline $\mathrm{ICH}$ scores & & & $0.007(0.004,0.010)$ & $<0.001$ & & $0.295(0.143,0.446)$ & $<0.001$ \\
\hline 0 & $90(0.44)$ & $0.970 \pm 0.072$ & & & $79.27 \pm 13.06$ & & \\
\hline 1 & 709 (3.43) & $0.951 \pm 0.128$ & & & $78.67 \pm 13.82$ & & \\
\hline 2 & 2,639 (12.76) & $0.954 \pm 0.108$ & & & $78.35 \pm 14.45$ & & \\
\hline 3 & $4,914(23.76)$ & $0.956 \pm 0.107$ & & & $78.81 \pm 14.22$ & & \\
\hline 4 & $5,506(26.62)$ & $0.961 \pm 0.090$ & & & $79.71 \pm 14.06$ & & \\
\hline 5 & $4,518(21.84)$ & $0.967 \pm 0.084$ & & & $80.15 \pm 13.75$ & & \\
\hline 6 & $2,297(11.11)$ & $0.9726 \pm 0.068$ & & & $80.96 \pm 13.44$ & & \\
\hline 7 & $10(0.05)$ & $0.9733 \pm 0.045$ & & & $79.80 \pm 13.84$ & & \\
\hline \multicolumn{8}{|l|}{$\mathrm{ICH}$ group } \\
\hline Ideal CVH & 6,825 (33.00) & $0.969 \pm 0.079$ & 0 & & $80.43 \pm 13.65$ & 1 & \\
\hline Intermediate $\mathrm{CVH}$ & $10,420(50.38)$ & $0.959 \pm 0.099$ & $0.016(0.005,0.027)$ & 0.004 & $79.28 \pm 14.14$ & $1.110(0.574,1.646)$ & $<0.001$ \\
\hline Poor CVH & $3,438(16.62)$ & $0.954 \pm 0.111$ & $0.027(0.015,0.040)$ & $<0.001$ & $78.44 \pm 14.29$ & $1.193(0.600,1.787)$ & $<0.001$ \\
\hline
\end{tabular}

Utility scores: The European Quality of Life Five Dimension Five Level Scale utility scores; VAS scores: Visual analogue scale scores; IHB scores, Ideal health behaviors scores; IHF scores, Ideal health factors cores; ICH scores, ideal cardiovascular health scores; Poor CVH, poor cardiovascular health (0-2 of ideal cardiovascular health scores); Intermediate CVH, intermediate cardiovascular health (three to four of ideal cardiovascular health scores); Ideal $\mathrm{CVH}$, ideal cardiovascular health (five to seven of ideal cardiovascular health scores).

${ }^{a}$ Multivariate Tobit regression model.

${ }^{b}$ Generalized linear model.

dimension of was the least report $(0.91 \%)$. In these three dimensions (mobility, self-care and usual activities), the proportion of participants with ideal $\mathrm{CVH}$ with problems was lower than that of participants with non-ideal CVH. Except for the pain/discomfort dimensions, other four dimensions were all significantly different among ICH groups (all $p<0.001$ ). 


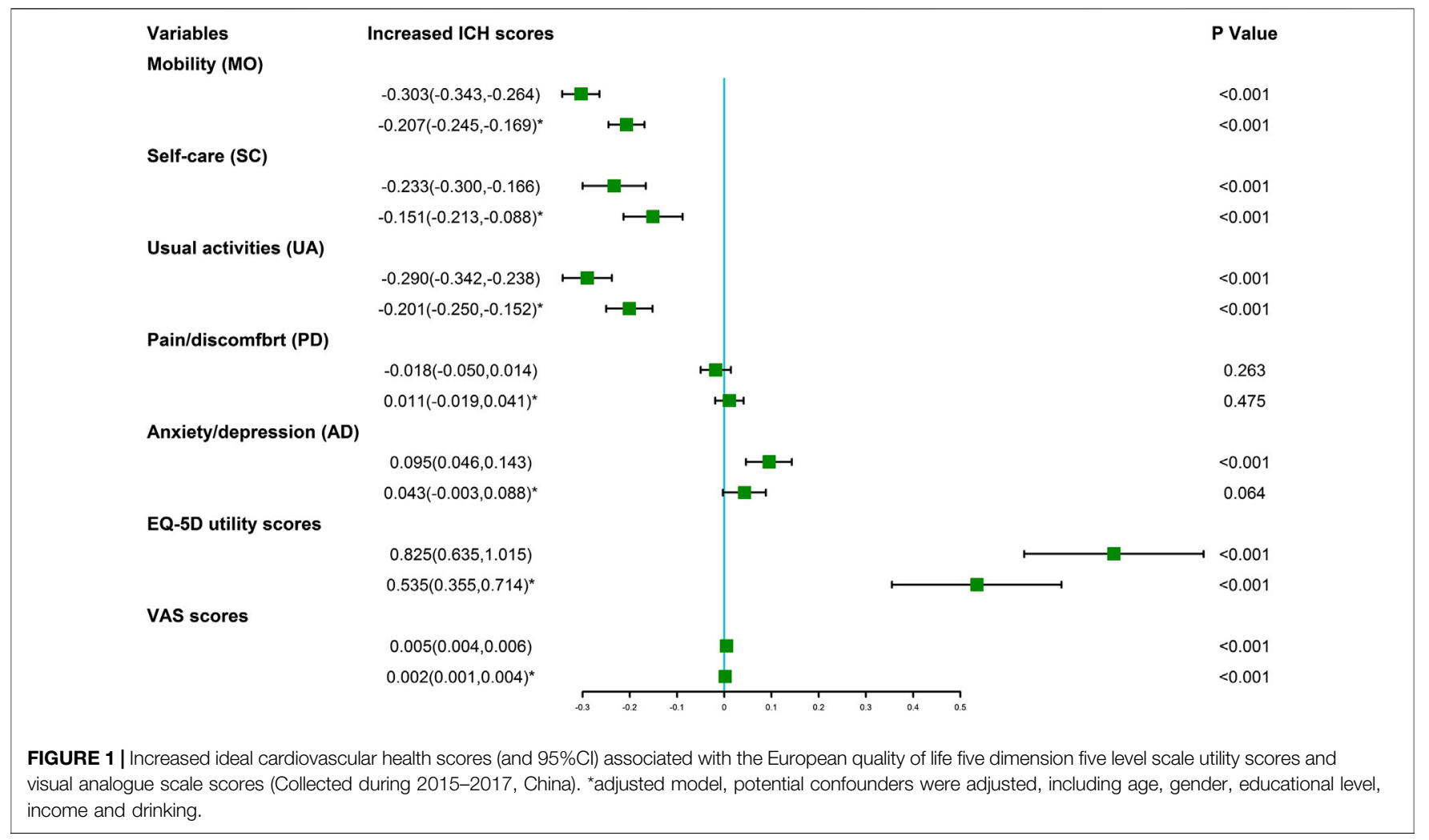

\section{Associations Between Ideal Cardiovascular Health and Health-Related Quality of Life}

Table 3 shows the association between ICH metrics and HRQoL. Ideal total cholesterol, ideal fasting plasma glucose and ideal physical activity were positively related with EQ-5D utility scores and VAS scores, respectively. In addition, IHB scores, IHF scores and ICH scores were positively related with EQ-5D utility scores and VAS scores, respectively (Supplementary Table S1). The results of crude linear regression models showed higher ICH scores was associated with increment of EQ-5D utility scores and VAS scores. After adjusted for potential confounders, the ICH scores increased associated with increment of EQ-5D utility scores and VAS scores were $0.535(0.355,0.714)$ and 0.002 $(0.001,0.004)$, respectively (Figure 1). The results of adjusted logistic regression models showed that the odds ratios and 95\% CIs of ideal ICH associated with increment of EQ-5D utility scores and VAS scores were $0.502(0.350,0.718)$ and $0.998(0.996$, 1.001), respectively (Figure 2).

\section{Interaction Analyses of Ideal Cardiovascular Health and Health-Related Quality of Life}

In the interaction analyses, it revealed that the association between $\mathrm{ICH}$ and HRQoL was modified by age, gender, income and educational lever and drinking in Supplementary Table S2. Stronger associations between ICH and EQ-5D utility scores were presented among women participants, those aged $\geq 60$ years, with low income, low education level and non-drinker participants. However, there was an significantly positive correlation between $\mathrm{ICH}$ and VAS scores, and stronger associations between ICH and VAS scores were presented among men, those aged $<40$ years, with low income, high education level and drinker.

\section{DISCUSSION}

To the best of our knowledge, this is the first study to examine the effect of HRQoL on ideal cardiovascular health (ICH) use the European Quality of Life Five Dimension Five Level Scale (EQ5D-5L) among rural adults. Based on the baseline survey of the Henan Rural Cohort, participants' cardiovascular health was evaluated via $\mathrm{ICH}$ scores and their HRQoL were estimated by EQ-5D-5L. The EQ-5D utility scores and VAS scores of participants with intermediate and ideal $\mathrm{CVH}$ were higher than those poor $\mathrm{CVH}$. ICH scores were positively correlated with EQ-5D utility scores and VAS scores, respectively. Stronger associations between ICH and EQ-5D utility scores were presented among women participants, those aged $\geq 60$ years, low income, low education level and non-drinker. However, stronger associations between ICH and VAS scores were presented among men, those aged $<40$ years, low income, high education level and drinker. In addition, we further conclude that HRQoL was the influencing factor of ideal cardiovascular health.

Prospective studies consistently demonstrated that individuals with a higher number of ideal cardiovascular health metrics have lower risks of hypertension [4], type 2 diabetes mellitus [5], CVD 


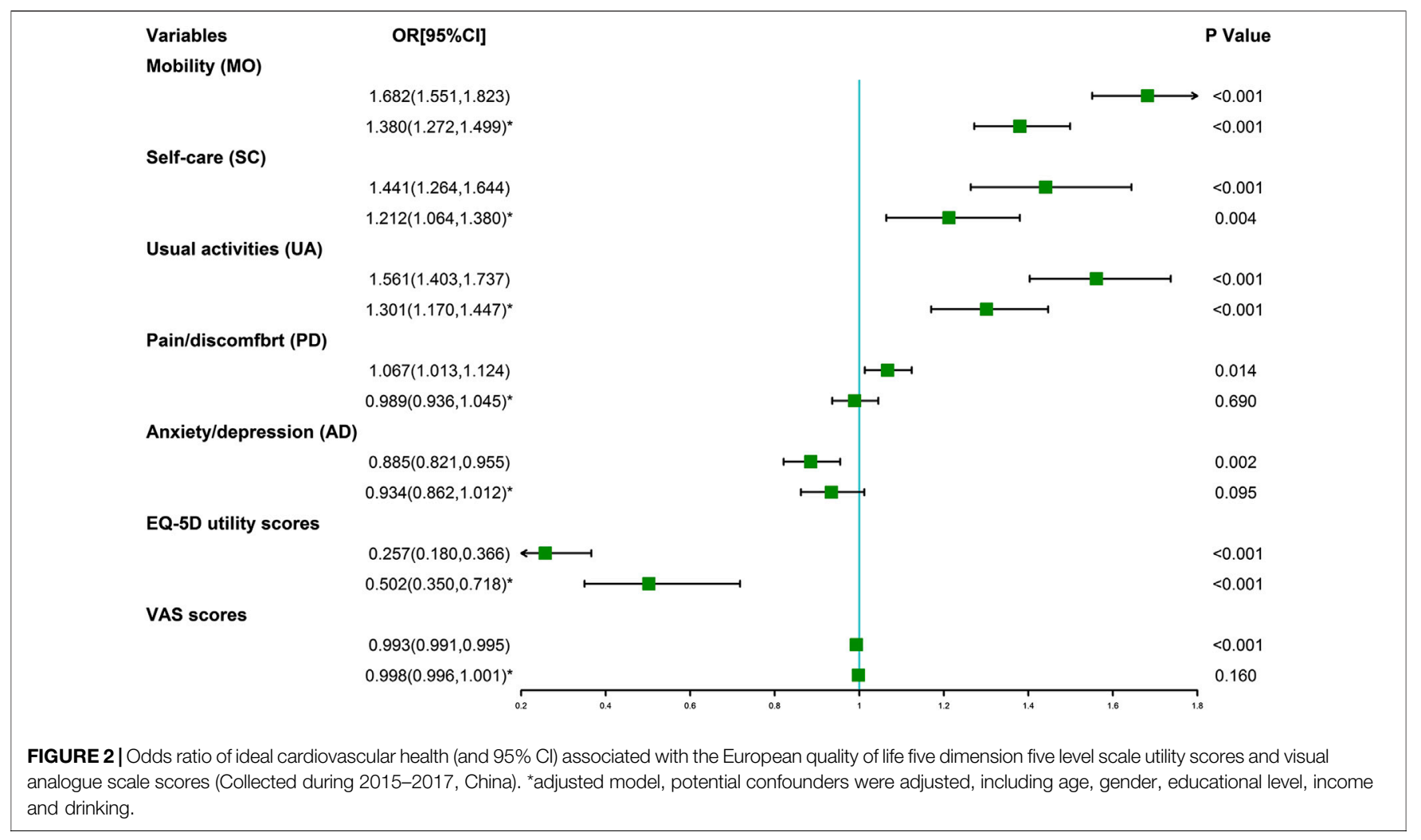

events (stroke, heart failure, myocardial infarction, and fatal coronary disease) [6, 7], and all-cause mortality [4]. Our results extend these findings providing evidence by demonstrating the possible beneficial effect of cardiovascular health on HRQoL. People with cardiovascular conditions or diabetes had higher risk of reporting poor HRQoL outcomes [21]. A greater number of multiple CVD risk factors may be associated with more detrimental impairment of HRQoL [22]. A recent study from the National Health and Nutrition Examination Survey, showed that compared to those with poor $\mathrm{CVH}$, individuals in intermediate and ideal $\mathrm{CVH}$ were 44 and $71 \%$ less likely to report being in fair/poor health [12]. Another study of the Coronary Artery Risk Development in Young Adults study reported that $\mathrm{t}$ maintaining ideal $\mathrm{CVH}$ from early adulthood results in higher health-related quality of life in middle age [13].

HRQoL was considered both a risk factor for and consequence of low levels of $\mathrm{CVH}$ [23]. Previous studies have conceptualized lower self-rated health as a risk factor for low CVH $[12,14]$. Similar results were found in our study, which was illustrated that EQ-5D utility scores and VAS scores were associated with increased ICH scores and higher risk of ideal CVH. Our study further found that ideal health behaviors and ideal health factors were positively correlated with EQ-5D utility scores and VAS scores, respectively. A review showed cross-sectional data showed a consistently positive association between physical activity level and health-related quality of life [11]. Physical activity of recommending levels were associated with better HRQoL [24]. A study reaffirms the significant association between smoking and HRQoL in a large nationally representative sample [25]. Adults with the metabolic syndrome experience worse health-related quality of life than adults without this syndrome [10]. Patients with longer duration of hypertension scored lower on HRQoL than others [26]. Dyslipidaemia was correlated with a lower HRQoL [27]. Thus, health behaviors and health factors included in the definition of overall cardiovascular health represents an important step toward to improve the HRQoL status, and it is urgent to promote status on smoking, diet and blood pressure.

In the interaction analyses, it revealed that the association between ICH and HRQoL was modified by age, gender, income and educational level and drinking. Stronger effects of ICH on HRQoL were observed among those aged $\geq 60$ years, low income and non-drinking. For different ages, genders and different social factors, our findings make us think about possible interventions to improve HRQoL. The oldest old population has become the fastest growing segment who have excess need of care and social support. Quality of life was not only associated with age-related diseases, but also correlated with a range of health-related lifestyles, and factors indicating social and family support [28]. it is crucial to improve the health-related quality of life (HRQoL) of these populations. Previous study reported the relationship between socio-economic status and health [29]. As the largest developing country, China is experiencing rapidly growing income inequality. To improve people's quality of life, it is important to ameliorate their socio-economic status, especially in rural area. Drinking habits showed lower physical healthrelated quality of life scores [30]. There was a negative correlation between alcohol consumption and health related quality of life [31]. Thus, limiting alcohol consumption may increase the 
correlation between cardiovascular health and health-related quality of life.

\section{Strengths and Limitations}

Our research has some advantages. Firstly, compared with the previous research $[12,14]$, we used EQ-5D-5L for the first time to evaluate the association between ideal cardiovascular health and health-related quality of life of Chinese rural population, bringing less ceiling effect [9]. secondly, we applied the newly developed EQ-5D-5L value set [19] based on Chinese population to Chinese rural population for the first time, avoiding bias due to cultural and population discrepancies compared with the value set based on other countries. In addition, most of the research objects are middle-aged and elderly people, which can better represent the current structure of Chinese rural population. Therefore, our findings can be extended to all rural areas in China. Nevertheless, several limitations should also be considered. Firstly, this is a crosssectional study, thus do not accurately describe causality. Secondly, some residents, such as college students and migrant workers, were not included in the scope of this study because they were studying or working outside. These people are more likely to be young and healthy and have a higher prevalence of ICH, which may lead to the underestimation of $\mathrm{ICH}$ in the rural population. Finally, the EQ-5D-5L value set for China was developed from urban areas rather than rural areas, which may introduce bias to our study.

\section{CONCLUSION}

In rural China, achieving better cardiovascular health metrics is associated with better HRQoL, which may extend the benefits of improving cardiovascular health beyond reducing the incidence of CVD and disability. Improvement of cardiovascular health may help to improve HRQLL among rural population of China.

\section{DATA AVAILABILITY STATEMENT}

The raw data supporting the conclusions of this article will be made available by the authors, without undue reservation.

\section{REFERENCES}

1. GBD 2017 Causes of Death Collaborators. Global regional, and national agesex-specific mortality for 282 causes of death in 195 countries and territories, 1980-2017: a systematic analysis for the Global Burden of Disease Study 2017. Lancet (2018). 392(10159):1736-88. doi:10.1016/S0140-6736(18) 32203-7

2. GBD 2017 DALYs and Hale Collaborators. Global regional, and national disability-adjusted life-years (DALYs) for 359 diseases and injuries and healthy life expectancy (HALE) for 195 countries and territories, 1990-2017: a systematic analysis for the Global Burden of Disease Study 2017. Lancet (2018). 392(10159):1859-922. doi:10.1016/S0140-6736(18)32335-3

3. Lloyd-Jones DM, Hong Y, Labarthe D, Mozaffarian D, Appel LJ, Van Horn L, et al. Defining and setting national Goals for cardiovascular health promotion

\section{ETHICS STATEMENT}

The studies involving human participants were reviewed and approved by the Zhengzhou University Life Science Ethics Committee (code [2015] MEC (S128)). The patients/participants provided their written informed consent to participate in this study.

\section{AUTHOR CONTRIBUTIONS}

During the research, YL conceived and designed the study. XW, YL had full access to all the data in the study. XW, YL analyzed the data and wrote the manuscript. TA corrected the manuscript. XW, XL, WL, NK, SS, TA, MN, YH, ZZ, MP, CW, YL conducted the collection of the data. All authors read and approve this version of the article.

\section{FUNDING}

This research was supported by the Foundation of National Key Program of Research and Development of China (Grant NO: 2016YFC0900803), The Science and Technology Innovation Team Support Plan of Colleges and Universities in Henan Province (Grant NO:21IRTSTHN029), National Natural Science Foundation of China (Grant NO: 81930092, 81573243, 81602925), Henan Natural Science Foundation (Grant NO: 182300410293), Discipline Key Research and Development Program of Zhengzhou University (Grant NO: XKZDQY202008, XKZDQY202002).

\section{CONFLICT OF INTEREST}

The authors declare that the research was conducted in the absence of any commercial or financial relationships that could be construed as a potential conflict of interest.

\section{SUPPLEMENTARY MATERIAL}

The Supplementary Material for this article can be found online at: https://www.ssph-journal.org/articles/10.3389/ijph.2021.592043/ full\#supplementary-material.

and disease reduction. Circulation (2010). 121(4):586-613. doi:10.1161/ circulationaha.109.192703

4. Corlin L, Short MI, Vasan RS, and Xanthakis V. Association of the duration of ideal cardiovascular health through adulthood with cardiometabolic outcomes and mortality in the framingham offspring study. JAMA Cardiol (2020). 5(5): 549-56. doi:10.1001/jamacardio.2020.0109

5. Joseph JJ, Bennett A, Echouffo Tcheugui JB, Effoe VS, Odei JB, Hidalgo B, et al. Ideal cardiovascular health, glycaemic status and incident type 2 diabetes mellitus: the REasons for Geographic and Racial Differences in Stroke (REGARDS) study. Diabetologia (2019). 62(3):426-37. doi:10.1007/s00125018-4792-y

6. Zhou L, Zhao L, Wu Y, Wu Y, Gao X, Li Y, et al. Ideal cardiovascular health metrics and its association with 20-year cardiovascular morbidity and mortality in a Chinese population. J Epidemiol Community Health (2018). 72(8):752-8. doi:10.1136/jech-2017-210396 
7. Folsom AR, Yatsuya H, Nettleton JA, Lutsey PL, Cushman M, and Rosamond WD. Community prevalence of ideal cardiovascular health, by the American Heart Association definition, and relationship with cardiovascular disease incidence. J Am Coll Cardiol (2011). 57(16):1690-6. doi:10.1016/j.jacc.2010. 11.041

8. Karimi M, and Brazier J. Health, health-related quality of life, and quality of life: what is the difference?. Pharmacoeconomics (2016). 34(7):645-9. doi:10.1007/ s40273-016-0389-9

9. Cunillera O, Tresserras R, Rajmil L, Vilagut G, Brugulat P, Herdman M, et al. Discriminative capacity of the EQ-5D, SF-6D, and SF-12 as measures of health status in population health survey. Qual Life Res (2010). 19(6):853-64. doi:10. 1007/s11136-010-9639-z

10. Ford ES, and Li C. Metabolic syndrome and health-related quality of life among U.S. adults. Ann Epidemiol (2008). 18(3):165-71. doi:10.1016/j. annepidem.2007.10.009

11. Bize R, Johnson JA, and Plotnikoff RC. Physical activity level and healthrelated quality of life in the general adult population: a systematic review. Prev Med (2007). 45(6):401-15. doi:10.1016/j.ypmed.2007.07.017

12. Allen NB, Badon S, Greenlund KJ, Huffman M, Hong Y, and Lloyd-Jones DM. The association between cardiovascular health and health-related quality of life and health status measures among U.S. adults: a cross-sectional study of the National Health and Nutrition Examination Surveys, 2001-2010. Health Qual Life Outcomes (2015). 13:152. doi:10.1186/s12955-015-0352-z

13. Pool LR, Ning H, Huffman MD, Reis JP, Lloyd-Jones DM, and Allen NB. Association of cardiovascular health through early adulthood and healthrelated quality of life in middle age: the Coronary Artery Risk Development in Young Adults (CARDIA) Study. Prev Med (2019). 126:105772. doi:10.1016/j. ypmed.2019.105772

14. Odom EC, Fang J, Zack M, Moore L, and Loustalot F. Associations between cardiovascular health and health-related quality of life, behavioral risk factor surveillance system, 2013. Prev Chronic Dis (2016). 13:E99. doi:10.5888/pcd13. 160073

15. Liu X, Mao Z, Li Y, Wu W, Zhang X, Huo W, et al. Cohort Profile: the Henan Rural Cohort: a prospective study of chronic non-communicable diseases. Int J Epidemiol (2019). 48:1756. doi:10.1093/ije/dyz039

16. Bauman A, Bull F, Chey T, Craig CL, Ainsworth BE, Sallis JF, et al. The international prevalence study on physical activity: results from 20 countries. Int J Behav Nutr Phys Act (2009). 6:21. doi:10.1186/1479-5868-6-21

17. Xue Y, Yang K, Wang B, Liu C, Mao Z, Yu S, et al. Reproducibility and validity of an FFQ in the henan rural cohort study. Public Health Nutr (2019). 23: 34-40. doi:10.1017/s1368980019002416

18. Han C, Liu F, Yang X, Chen J, Li J, Cao J, et al. Ideal cardiovascular health and incidence of atherosclerotic cardiovascular disease among Chinese adults: the China-PAR project. Sci China Life Sci (2018). 61(5):504-14. doi:10.1007/ s11427-018-9281-6

19. Luo N, Liu G, Li M, Guan H, Jin X, and Rand-Hendriksen K. Estimating an EQ-5D-5L value set for China. Value in Health (2017). 20(4):662-9. doi:10. 1016/j.jval.2016.11.016
20. Austin PC. Bayesian extensions of the Tobit model for analyzing measures of health status. Med Decis Making (2002). 22(2):152-62. doi:10.1177/ 0272989X0202200212

21. Chen HY, Baumgardner DJ, and Rice JP, Health-related quality of life among adults with multiple chronic conditions in the United States, Behavioral Risk Factor Surveillance System, 2007. Prev Chronic Dis (2011). 8(1):A09.

22. Li C, Ford ES, Mokdad AH, Balluz LS, Brown DW, and Giles WH. Clustering of cardiovascular disease risk factors and health-related quality of life among US adults. Value in Health (2008). 11(4):689-99. doi:10.1111/j.1524-4733.2007.00307.x

23. Rumsfeld JS, Alexander KP, Goff DC, Graham MM, Ho PM, Masoudi FA, et al. Cardiovascular health: the importance of measuring patient-reported health status. Circulation (2013). 127(22):2233-49. doi:10.1161/CIR. 0b013e3182949a2e

24. Brown DW, Balluz LS, Heath GW, Moriarty DG, Ford ES, Giles WH, et al. Associations between recommended levels of physical activity and healthrelated quality of life Findings from the 2001 Behavioral Risk Factor Surveillance System (BRFSS) survey. Prev Med (2003). 37(5):520-8. doi:10. 1016/s0091-7435(03)00179-8

25. Mody RR, and Smith MJ. Smoking status and health-related quality of life: findings from the 2001 behavioral risk factor surveillance system data. Am $J$ Health Promot (2006). 20(4):251-8. doi:10.4278/0890-1171-20.4.251

26. Liang Z, Zhang T, Lin T, Liu L, Wang B, Fu AZ, et al. Health-related quality of life among rural men and women with hypertension: assessment by the EQ5D-5L in Jiangsu, China. Qual Life Res (2019). 28(8):2069-80. doi:10.1007/ s11136-019-02139-3

27. Wu H, Li H, Li H, Ding Y, Wang C, Zhang G, et al. Evaluation of health-related quality of life in adults with and without dyslipidaemia in rural areas of central China. Qual Life Res (2020). 29(4):925-39. doi:10.1007/s11136-019-02336-0

28. Chen C, Liu GG, Shi QL, Sun Y, Zhang H, Wang MJ, et al. Health-related quality of life and associated factors among oldest-old in China. J Nutr Health Aging (2020). 24(3):330-8. doi:10.1007/s12603-020-1327-2

29. Kondo N, Sembajwe G, Kawachi I, van Dam RM, Subramanian SV, and Yamagata Z. Income inequality, mortality, and self rated health: meta-analysis of multilevel studies. Bmj (2009). 339:b4471. doi:10.1136/bmj.b4471

30. Wang Y, Huang L, and Zhou L-X Correlation between exercise, personal income level and health-related quality of life in patients with newly diagnosed stable angina. Mil Med Res (2019). 6(1):36. doi:10.1186/s40779-019-0226-5

31. Wu J, Han Y, Xu J, Lu Y, Cong H, Zheng J, et al. Chronic stable angina is associated with lower health-related quality of life: evidence from Chinese patients. PLoS One (2014). 9(5):e97294. doi:10.1371/journal.pone.0097294

Copyright (c) 2021 Wu, Liu, Liao, Kang, Sang, Abdulai, Niu, He, Zhai, Pan, Wang and Li. This is an open-access article distributed under the terms of the Creative Commons Attribution License (CC BY). The use, distribution or reproduction in other forums is permitted, provided the original author(s) and the copyright owner(s) are credited and that the original publication in this journal is cited, in accordance with accepted academic practice. No use, distribution or reproduction is permitted which does not comply with these terms. 